\title{
SYMMETRY VIOLATION IN HEAVY NUCLEI
}

\author{
VLADIMIR GUDKOV \\ Department of Physics and Astronomy, University of South Carolina, Columbia \\ SC 29208, USA \\ E-mail: gudkov@sc.edu
}

\begin{abstract}
The advantage of searching for violation of Time Reversal Invariance in neutron induced reactions using relative measurements of Time and Parity violating effects is discussed. This approach gives the enhancement of $T$-violating effects by many orders of magnitude and, at the same time, decrees theoretical uncertainties and experimental errors.
\end{abstract}

\section{Introduction}

For a long time heavy nuclei were considered for the study of symmetry violations mostly due to enhancement factors related to the complex nuclear structure. Classical examples are experiments that measure parity violating correlations in nuclear decaysand in nuclear reactions. It is well known that due to nuclear enhancements] the parity violating effects in nuclei could be as large as $\sim 10^{-1}$ in comparison to its "natural" scale $\sim 10^{-7}$ for the simplest few nucleon systems. This feature of heavy nuclei has been explored for the gathering of unique information about statistical properties of nuclei and for further dejelement of the understanding of nuclear structure (see, for example ref. 4.6 . 6 . However, it is practically impossible to obtain information about weak interactions using these precisely measured nuclear $P$-violating effects. The main reason for that is the extremely complicated structure of nuclear wave functions of the excited states of heavy nuclei. Therefore, $P$ violating effects in heavy nuclei could be treated by using mainly statistical approaches.

Time Reversal Invariance (TRI) is another example of a fundamental symmetry that could be enhanced in heavy nuclei. Since the origin of the possible $T R I$ violation is not yet understood, and even a proof of the TRI violation ( $T$-violation) does not exist, it is very important to search for $T$ violation using all opportunities. To avoid a possible misunderstanding, we clarify the meaning of the proof of $T$-violation.

From the point of view of a local Lorentz invariant field theory with ordinary relations between spin and statistics, $T$-violation can be related through the $C P T$ theorem to $C P$-violation which was discovered experimentally in the $K^{0}$-meson decays. This kind of $T$-violating interaction violates parity, 
too. In this framework there is another possibility for $T$-violation due to $T$-riolating $P$-conserving $(C$-odd and $P$-even) interactions (see, for example ref $\mathrm{A}$ ). However, an extension of the theory beyond the locality of the interactions or with violation of Lorentz invariance and/or the spin-statistics relations might violate the $C P T$ theorem. Therefore, the CPLEAR experimental result could be considered as evidence of $T$-violation in $K^{0}$-meson decays under the assumption of the $C P T$ invariance.

It should be noted that in $K^{0}$-meson decays the $C P$-violating interactions belong to the $\Delta S=1$ sector. However, some models (for example, Minimal Super Symmetry Model) predict large $C P$-violating effects for the $\Delta S=0$ sector and a suppression of $C P$ violation with a change of strangeness $\Delta S=1$. From this point of view a low energy physics $(\Delta S=0)$ is very interesting because it can give independent additional information about $C P$ violation.

\section{$2 \quad T$-violation and neutron reactions}

Taking into account the above arguments one can see the importance of the search for $T$-violation in low energy physics. It equally applies to both nuclear reactions and measurements of neutron and atomic dipole moments. However, there are some specific features, both theoretical and experimental, which distinguish neutron induced reactions from a wide class of low energy experiments. Let us consider some of them.

The important questions to address in the search for the $T$-violation effects are: how well can the effect be calculated, and how well do we know existing restrictions on the $T$-violating coupling constants? These questions are very important for the interpretation of a positive experimental result. However, they are much more important for estimating the upper limit on the unknown $T$-violating constants in case the experiment will give only an upper bound on the value of the effect. In that relation, one recalls a history of calculations of the electric dipole moment $(E D M)$ for a simple system like the neutron. We can see that even for rather well defined mechanisms of $C P$ violation, such as the standard Kobayashi-Maskawa model and Weinberg model of spontaneous $C P$-violation in Higgs sector, the precisely estimated value had changed over time by many orders of magnitude. The reason for that is a discovery from time to time of different contributions from strong interactions (such a strange quark chromoelectric dipole moment or multi gluon $C P$-odd operator) which change drastically the estimated values of the neutron $E D M$. The situation could be much worse for more complicated systems. One of the recent examples of possibly similar difficulties is the discrepancy (by about $2.5 \sigma$ ) between the most precise measurement 12 of $P$ violation in the atom of

svhn: submitted to World Scientific on November 4, 2018 
${ }^{133} \mathrm{Cs}$ and its accurate theoretical calculations. This situation rises questions about a possible discovery of "new physics" or, probably, about simply an overestimation of the calculated accuracy.

Is it possible to avoid such difficulties in the search for an unknown phenomena like $T$ violation? Fortunately, for some cases of neutron induced reactions the answer is yes. The reasons for that are structural similarities between interactions with different symmetries and the possibility to measure different effects corresponding to a violation of these symmetries simultaneously. To illustrate the general idea, let us consider different types of interactions in the matrix momentum representation. Then, $P$ - and $T$-conserving strong interactions can be represented by an orthogonal (real symmetric) matrix:

$$
\mathbf{V}_{\mathbf{s t r}}=\left(\begin{array}{ccccc}
v_{00} & 0 & \ldots & v_{i k} & \ldots \\
0 & v_{11} & \ldots & \ldots & \ldots \\
\vdots & \vdots & \ddots & \ldots & \ldots \\
\vdots & v_{k i} & \vdots & v_{i i} & \ldots \\
\vdots & \vdots & \vdots & \vdots & \ddots
\end{array}\right)
$$

with $v_{i k}=0$ for angular momenta $i$ and $k$ with different parities.

In the same representation, parity violating interactions look like

$$
\mathbf{V}_{\mathbf{P}}=\left(\begin{array}{ccccc}
0 & v_{01} & \ldots & v_{i k} & \ldots \\
v_{10} & 0 & \ldots & \ldots & \ldots \\
\vdots & \vdots & \ddots & \ldots & \ldots \\
\vdots & v_{k i} & \vdots & 0 & \ldots \\
\vdots & \vdots & \vdots & \vdots & \ddots
\end{array}\right)
$$

with $v_{i k}=0$ for angular momenta $i$ and $k$ with the same parities. In this representation $T$-violating interactions bring anti-symmetric imaginary parts to all matrix elements. Therefore, $T$-violating $P$-conserving interactions are

$$
\mathbf{V}_{\mathbf{T}}=\left(\begin{array}{ccccc}
0 & 0 & \ldots & +i w_{i k} & \ldots \\
0 & 0 & \ldots & \ldots & \ldots \\
\vdots & \vdots & \ddots & \ldots & \ldots \\
\vdots & -i w_{k i} & \vdots & 0 & \ldots \\
\vdots & \vdots & \vdots & \vdots & \ddots
\end{array}\right)
$$


and $T$-violating $P$-violating interactions are

$$
\mathbf{V}_{\mathbf{P T}}=\left(\begin{array}{ccccc}
0 & +i w_{01} & \ldots & +i w_{i k} & \ldots \\
-i w_{10} & 0 & \ldots & \ldots & \ldots \\
\vdots & \vdots & \ddots & \ldots & \ldots \\
\vdots & -i w_{k i} & \vdots & 0 & \ldots \\
\vdots & \vdots & \vdots & \vdots & \ddots
\end{array}\right)
$$

One can see the similarity in the structure of the interactions with the same parity properties and different Time Reversal symmetries: $T$-violation brings only an asymmetric phase. However, the parity violation changes structure of the interactions dramatically. Therefore, we can avoid the influence of nuclear structure on the measurable parameter by choosing it as an appropriate ratio of $T$-violating and $T$-conserving effects. The example for one of the possible choices will be considered in next section.

Prior to that, let us recall a general experimental advantage of neutron reactions - extremely high energy resolution. This property gives us the opportunity to resolve very narrow resonances and to profit from the resonance enhancement factor in the search for $T$ violation. The typical neutron energy for the resonance reactions is about $1 \mathrm{eV}-100 \mathrm{eV}$. When neutron is captured by nucleus, the compound nuclear excitation energy is about $6 \mathrm{MeV}$. Then, without any effort the energy resolution is about $2 \cdot 10^{-7}$. It could be even much better in real experiments (by about $\sim 10^{-11}$ ). Taking into account the high experimentally achieved level of neutron polarization and the high flux with good time structure at the Spallation Neutron Source, one can conclude that neutron reactions are very promising for precise measurements of $T$ violation.

\section{$3 \quad P$ - and $T$ - violating effects in neutron scattering}

To illustrate the advantage of the search for $T$-violation in heavy nuclei with neutrons, we consider the case of simultaneous Parity and Time Reversal (PT) violation. One of the effects related to $P T$ violation is the difference of total cross sections $\Delta \sigma_{P T}$ in the transmission of polarized neutrons through a polarized target for opposite neutron spin orientations (For some other mechanisms of $T$ violation and other effects, see the paper $\$$ and references therein. The experimental proposal to search for $P T$ violation in a different geometry is discussed in the paper.11) This effect is proportional to the T-odd correlation $(\vec{\sigma} \cdot[\vec{k} \times \vec{I}])$ between spin $\vec{\sigma}$ and momentum $\vec{k}$ of neutron and nuclear spin $\vec{I}$. Let us also consider the corresponding $P$-violating $T$-conserving difference

svhn: submitted to World Scientific on November 4, 2018 
of total cross sections $\Delta \sigma_{P}$ in the transmission of polarized neutrons through unpolarized target which is proportional to the correlation $(\vec{\sigma} \cdot \vec{k})$. Using the optical theorem, one can represent these differences of total cross sections in terms of differences of zero angle scattering elastic amplitudes for opposite neutron spin orientations along axis $[\vec{k} \times \vec{I}]$ for $P T$-odd effect and along $\vec{k}$ for $P$-odd effect :

$$
\Delta \sigma_{P T}=\frac{4 \pi}{k} \operatorname{Im}\left(f_{\uparrow}-f_{\downarrow}\right)
$$

and

$$
\Delta \sigma_{P}=\frac{4 \pi}{k} \operatorname{Im}\left(f_{-}-f_{+}\right)
$$

One can calculate both these parameters using distorted wave Born approximation in the first power of parity and time reversal violating interactions (see, for example ref.2). Then the symmetry violating amplitudes can be written as

$$
t_{P, P T}^{f i}=<\Psi_{f}^{-}\left|V_{P, P T}\right| \Psi_{i}^{+}>
$$

where $\Psi_{i, f}^{ \pm}$are the eigenfunctions of the nuclear T-invariant Hamiltonian with the appropriate boundary conditions:

$$
\Psi_{i, f}^{ \pm}=\sum_{k} a_{k(i, f)}^{ \pm}(E) \phi_{k}+\sum_{m} \int b_{m(i, f)}^{ \pm}\left(E, E^{\prime}\right) \chi_{m}^{ \pm}\left(E^{\prime}\right) d E^{\prime} .
$$

Here $\phi_{k}$ is the wave function of the $k^{t h}$ compound-resonance and $\chi_{m}^{ \pm}(E)$ is the potential scattering wave function in the channel $m$. The coefficient

$$
a_{k(i, f)}^{ \pm}(E)=\frac{\exp \left( \pm i \delta_{i, f}\right)}{(2 \pi)^{\frac{1}{2}}} \frac{\left(\Gamma_{k}^{i, f}\right)^{\frac{1}{2}}}{E-E_{k} \pm \frac{i}{2} \Gamma_{k}}
$$

describes compound nuclear resonances reactions and the coefficient $b_{m(i, f)}^{ \pm}\left(E, E^{\prime}\right)$ describes potential scattering and interactions between the continuous spectrum and compound resonances. (Here $E_{k}, \Gamma_{k}$, and $\Gamma_{k}^{i}$ are the energy, the total width, and the partial width in the channel $i$ of the $k$-th nuclear compound resonance, $E$ is the neutron energy, and $\delta_{i}$ is the potential scattering phase in the channel $i ;\left(\Gamma_{k}^{i}\right)^{\frac{1}{2}}=(2 \pi)^{\frac{1}{2}}<\chi_{i}(E)|V| \phi_{k}>$, where $V$ is a residual interaction operator.)

Therefore, in general, there are many mechanisms that violate the symmetries in nuclei and theoretical descriptions of symmetry violating effects could be rather complicated. However, it was shown? (and confirmed by many experiments) that for the bulk of heavy nuclei the dominant mechanism for 
parity (and $P T$ ) violation is the mechanism of symmetry mixing on the compound nuclear stage. This mechanism could be described by the first term in Eq. (4). For our illustrative purposes we consider the simplest case of a two resonance approximation. This approximation is reasonably good for many heavy nuclei in the low neutron energy region $E \sim 1 \mathrm{eV}-10 \mathrm{eV}$, since the characteristic energy difference between compound neutron resonances with the same spin and parity is usually about $10 \mathrm{eV}-100 \mathrm{eV}$. Assuming that main effects could be described by mixing of the nearest $s$-wave and $p$-wave resonances, one derives the symmetry violating amplitudes as:

$$
<p|t| s>=-\frac{1}{2 \pi} \frac{(v+i w)\left(\Gamma_{s}^{n} \Gamma_{p}^{f}\right)^{\frac{1}{2}}}{\left(E-E_{s}+i \Gamma_{s} / 2\right)\left(E-E_{p}+i \Gamma_{p} / 2\right)} e^{i\left(\delta_{s}^{n}+\delta_{p}^{n}\right)}
$$

and

$$
<s|t| p>=-\frac{1}{2 \pi} \frac{(v-i w)\left(\Gamma_{p}^{n} \Gamma_{s}^{n}\right)^{\frac{1}{2}}}{\left(E-E_{s}+i \Gamma_{s} / 2\right)\left(E-E_{p}+i \Gamma_{p} / 2\right)} e^{i\left(\delta_{p}^{n}+\delta_{s}^{n}\right)} .
$$

The matrix elements $v$ and $w$ are real and correspond to the real and imaginary parts of the mixing matrix element between $s$ - and $p$-wave compound resonances for the sum of $P$ - and $P T$-violating operators $V_{P}$ and $V_{P T}$

$$
v+i w=<\phi_{p}\left|V_{P}+V_{P T}\right| \phi_{s}>.
$$

The matrix element $v$ violates parity but preserves Time Reversal Invariance. The matrix element $w$ violates both $P$ - and $T$-invariance. It contributes to the total mixing matrix element Eq. (8) as an imaginary anti-symmetric part which results in its opposite sign for the amplitudes in Eq. (6) and Eq. (7).

It is important that these amplitudes describe both $P$-violating and $P T$ violating processes. Moreover, matrix elements $v$ and $w$ in these amplitudes are matrix elements calculated using exactly the same wave functions.

Another remarkable fact is that the difference of amplitudes $\left(f_{-}-f_{+}\right)$ for $P$-violating effect in Eq. (2) is proportional to the sum of the symmetry violating amplitudes (Eq. (6) and Eq. (7)) but the difference of amplitudes $\left(f_{\uparrow}-f_{\downarrow}\right)$ for $P T$-violating effect in Eq. (11) is proportional to the difference of the same amplitudes (Eq. (6) and Eq. (7)). This results in the same energy dependencies for both $P$ - and $P T$-violating effects. Indeed, taking into account all numerical factors one gets:

$$
\Delta \sigma_{P T}=-\frac{2 \pi G_{J}}{k^{2}} \frac{w\left(\Gamma_{s}^{n} \Gamma_{p}^{n}(S)\right)^{\frac{1}{2}}}{[s][p]}\left[\left(E-E_{s}\right) \Gamma_{p}+\left(E-E_{p}\right) \Gamma_{s}\right],
$$


and

$$
\Delta \sigma_{P}=\frac{2 \pi G_{0}}{k^{2}} \frac{w\left(\Gamma_{s}^{n} \Gamma_{p}^{n}\right)^{\frac{1}{2}}}{[s][p]}\left[\left(E-E_{s}\right) \Gamma_{p}+\left(E-E_{p}\right) \Gamma_{s}\right],
$$

where $[s, p]=\left(E-E_{s, p}\right)^{2}+\Gamma_{s, p}^{2} / 4, G_{J}$ and $G_{0}$ are spin factors; $J$ and $S$ are compound nuclei and channel spins (see details in ref.2,218). Due to the similarity in these two parameters it is obvious that the $P T$-violating effect has the same resonance enhancement as the $P$-violating one. Also, they have similar dynamic enhancement factors. Therefore, one can conclude that the $P T$-violating effect has about the same nyclear enhancement factors $\left(\sim 10^{6}\right)$ as the $P$-violating one (see, for details ref. 22 ).

Taking the ratio of $\Delta \sigma_{P T}$ and $\Delta \sigma_{P}$ parameters from the same experiment at the same neutron energy one can extract the ratio of $P T$ - and $P$-violating matrix elements $\langle\lambda\rangle=w / v$ eliminating nuclear reaction uncertainties and experimental uncertainties related to the absolute normalization:

$$
\frac{\Delta \sigma_{C P}}{\Delta \sigma_{P}}=\kappa(J) \frac{w}{v}
$$

where $\kappa(J)$ is the calculable spin dependent coefficient 13

Now it is worthwhile to recall that the extracted ratio $\langle\lambda\rangle=w / v$ is not the ratio of independent matrix elements of complicated compound nuclear states, but rather the ratio of matrix elements with exactly the same wave functions and very similar operators 13 This ratio might be calculated for each particular nucleus with quite good accuracy. In the simplest case one can do it using one particle $P$-violating and $P T$-violating potentials

$$
\begin{gathered}
V_{P}=\frac{G}{8^{1 / 2} M}\{(\vec{\sigma} \cdot \vec{p}), \rho(\vec{r})\}_{+}, \\
V_{P T}=\frac{i G \lambda}{8^{1 / 2} M}\{(\vec{\sigma} \cdot \vec{p}), \rho(\vec{r})\}_{-}
\end{gathered}
$$

where $G$ is the weak interaction Fermi constant, $M$ is the proton mass, $\rho(\vec{r})$ is the nucleon density, $\vec{p}$ is the momentum of the valence nucleon, and $\lambda=$ $g_{P T} / g_{P}$ is the ratio of $P T$-violating to $P$-violating nucleon - nucleon coupling constants. Then one obtains

$$
<\lambda>=\frac{\lambda}{1+2 \xi}
$$

where $\xi \sim(1-7)$ (for detailed discussions see papers13, $4,155.16$ ).

Now we come to the result that the simultaneous measurement of $P T$ and $P$-violating effects affords the opportunity to extract the ratio of $P T$ - 
Table 1 . The relative values $\lambda$ of the $C P$-violating nucleon coupling constants.

\begin{tabular}{|c|l|}
\hline Model & $\lambda$ \\
\hline Kobayashi-Maskawa & $\leq 10^{-10}$ \\
Right-Left model & $\leq 4 \times 10^{-3}$ \\
Horizontal symmetry & $\leq 10^{-5}$ \\
Weinberg model (charged Higgs bosons) & $\leq 2 \times 10^{-6}$ \\
Weinberg model (neutral Higgs bosons) & $\leq 3 \times 10^{-4}$ \\
$\theta$-term in $Q C D$ & $\leq 5 \times 10^{-5}$ \\
Neutron EDM (one $\pi$-loop mechanism) & $\leq 4 \times 10^{-3}$ \\
Atomic EDM $\left({ }^{199} \mathrm{Hg}\right)$ & $\leq 2 \times 10^{-3}$ \\
\hline
\end{tabular}

and $P$-violating nucleon coupling constants, $\lambda=g_{P T} / g_{P}$, eliminating nuclear uncertainties and some experimental uncertainties.

Let us compare the accuracy for the parameter $\lambda_{\exp } \sim 10^{-4}$ which could be achieved 17 at the Spallation Neutron Source with theoretical expectations for the ratio $\lambda=g_{P T} / g_{P}$. To do this we assume that $P T$ violation is related to the $C P$ violation under the $C P T$-theorem. (All existing calculations for the parameter $\lambda$ have been done for the $C P T$-invariant models.) The estimated values 13.18 .19 .25 of the parameter $\lambda$ for some models of $C P$ violation are given in Table1. The last two rows in Table 1 correspond to limits on the parameter $\lambda$ obtained from the experimental results of measurements of neutron and atomic electric dipole moments. The parameter $\lambda$ of neutron electric dipole moment21 (EDM) has been calculated using phenomenological $\pi$-meson one loop mechanism The parameter $\lambda$ of atomic EDM $\left({ }^{199} \mathrm{Hg}\right) 22$ is addopted from the paper.23

It should be noted that due to model uncertainties and low energy $Q C D$ corrections, the reasonable accuracy of the above estimations is usually about one order of magnitude. Therefore, the comparison of the $\lambda_{\exp }$ and $\lambda$ presented in Table 1 leads to the observation that the possible experiment for the search for $T$ violation at the SNS has reasonably good sensitivity to test many models of $C P$ violation.

For the completeness of our discussion, it is necessarily to mention that a real experimental setup for these types of experiments should be quite complicated in order to eliminate the Final State Interactions (FSI) and to control them during the experiment. It is well known that a $T$-odd correlations have no relations to the Time Reversal Invariance (TRI) in general. This is different from the case of Parity Invariance. The reason for this difference is the fact that the Time Reversal operator, unlike the Parity operator, has no eigenstates nor eigenvalues. Therefore, it leads not to restrictions on the reaction amplitude but to a relation between amplitudes for two different processes.

svhn: submitted to World Scientific on November 4, 2018 
However, under special circumstances TRI could be related to $T$-odd correlations with some restrictions. The well known example is the $T$-odd correlations in nuclear (particle) decays when the decay can be described in the first Born approximation. In that case the unitarity condition of the scattering matrix leads to its hermicity, which is an additional condition on the amplitude resulting in the similarity between $T$-odd and $P$-odd operators and, as a consequence, in the connection of $T$-odd correlations to the $T$-violating interactions. When the other Born terms of higher order (usually called the FSI) become significant, the hermicity condition breaks down and the $T$-odd correlations could be produced without $T$-violating interactions.

In our case the neutron transmission is an elastic scattering at the zero angle. Then, the initial and final states in this process coincide. It gives us the additional condition on the scattering amplitude without any reference to the intensity of the interactions. Therefore, $T$-odd correlations in the elastic scattering always are related to $T$-violating interactions.

In the experiment there is a possibility of neutron depolarization due to the neutron spin precession in magnetic fields, strong spin-spin interactions, and weak interactions. All these effects could destroy the elasticity of the process. Fortunately, it has been shown that these interference effects can be decreased or compensated with a given accuracy, and after that, could be kept under control dyije the experiment. For detailed discussions of these problems see papers11, $24,25,26,27$ and references therein.

\section{Summary}

To summarize, the considered advantages of the search for $T$-violating effects in neutron reactions on heavy nuclei at the SNS, we emphases again that the unambiguous experimental results are guaranteed by the comparing the proper symmetry violating effects in the same experimental setup. It does not only give us the possibility to decrease the experimental uncertainties, but, what is much more important, it allows us to measure relative parameters which are almost free from usually unavoidable theoretical uncertainties. This feature, in the combination with the large nuclear enhancement $\left(\sim 10^{6}\right)$, and the possibility to get rid of final state interactions at any desirable level, gives us a unique opportunity to search for $T$ violation at the SNS. The $T$-violating effects in neutron reactions are a pure test of the $T$ violation with no reference to the $C P T$ theorem. It is also important because low energy processes test $\Delta S=0$ sector of the $C P$ interactions which is out of reach for high energy experiments.

svhn: submitted to World Scientific on November 4, 2018 


\section{References}

1. O.P. Sushkov and V.V. Flambaum, Sov.Phys.Usp. 25, 1 (1982).

2. V.E. Bunakov and V.P. Gudkov, Nucl.Phys. A401, 93 (1983).

3. V.P. Alfimenkov, Sov.Phys.Usp. 27, 797 (1984).

4. Y. Masuda et al., Nucl.Phys. A504, 269 (1989).

5. J.D. Bowman et al., Annu.Rev.Nucl.Part.Sci. 43, 829 (1993).

6. G. E. Mitchell, in this proceding.

7. CPLEAR Collaboration, Phys.Let. B444, 43 (1998).

8. V.P. Gudkov, Phys.Rep. 212, 77 (1992).

9. M.J. Ramsey-Musolf, in this proceding.

10. S.C. Bennett and C.E. Wieman, Phys. Rev. Lett. 82, 2484 (1999).

11. Y. Masuda, in this proceding.

12. V.E. Bunakov and V.P. Gudkov, Z.Phys. A308, 363 (1982).

13. V.P. Gudkov, Phys.Let. B243, 319 (1990).

14. I.S. Towner and A.C. Hayes, Phys.Rev. C49, 2391 (1994).

15. I.B. Khriplovich, Phys.Rev. C52, 1138 (1995).

16. B. Desplanques and S. Noguera, Nucl.Phys. A581, 1 (1995).

17. Y. Masuda, privaite communication.

18. P. Hercezg in Test of Time Reversal Invariance in Neutron Physics, eds. N.R. Roberson, C.R. Gould and J.D. Bowman (World Scientific, Singapore, 1987), p.24.

19. P. Hercezg in Parity and Time Reversal Violation in Compound Nuclear States and Related Topics, eds. N. Auerbach and J.D. Bowman (World Scientific, Singapore, 1996), p.214.

20. V.P. Gudkov in Parity and Time Reversal Violation in Compound Nuclear States and Related Topics, eds. N. Auerbach and J.D. Bowman (World Scientific, Singapore, 1996), p.231.

21. P.G. Harris et al., Phys.Rev.Let. 82, 904 (1999).

22. J.P. Jacob et al., Phys. Rev. Lett. 71, 3782 (1993).

23. T. Falk et al., Nucl.Phys. B560, 3 (1999).

24. V.E. Bunakov and V.P. Gudkov, J.Phys.(Paris) 45, C3-77 (1984).

25. V.P. Gudkov, Phys.Rev. C46, 357 (1992).

26. L. Stodolsky, Phys.Let. B212, 172 (1986).

27. P.K. Kabir, Phys.Rev. D37, 1856 (1988). 